\title{
An "alternative finger" in robotic-assisted thoracic surgery: intraoperative ultrasound localization of pulmonary nodules
}

\author{
Zhenyu Zhou' ${ }^{1}$, Zhitian Wang', Zhelan Zheng ${ }^{2}$, Jinlin Cao', Chong Zhang ${ }^{1}$, Zhehao He ${ }^{1}$, \\ Wang Lv', Jian Hu ${ }^{1}$
}

${ }^{1}$ Department of Thoracic Surgery, ${ }^{2}$ Department of Ultrasound Imaging, the First Affiliated Hospital, College of Medicine, Zhejiang University, Hangzhou, China

\begin{abstract}
Aim: Robotic-assisted thoracic surgery (RATS) has become a promising treatment for pulmonary neoplasms. During RATS, intraoperative ultrasonography can act as an "alternative finger" to "touch" and locate lesions, especially pulmonary nodules. This study was aimed to investigate the efficacy of intraoperative ultrasonographic localization during da Vinci RATS procedures. Material and methods: Patients with pulmonary nodules were randomly divided into an Experimental Group and Control Group in which nodules were respectively located using intraoperative ultrasonography or by the surgeon's anatomic knowledge. The success rates and relevant localization factors were compared between the groups and analyzed to conclude the efficacy of intraoperative ultrasonography. Additionally, the intraoperative ultrasonography learning curve was analyzed to evaluate each surgeon's ability to independently perform intraoperative ultrasonography. Results: Thirty-four patients were included in the study ( $\mathrm{n}=17$ /group). Respectively, the Experimental Group and Control Group comprised $41.2 \%$ and $58.9 \%$ women $(\mathrm{p}=0.937)$, and had average ages of 55.5 and 55.8 years. In the Experimental group, ultrasonographic localization for mixed ground-glass nodules with CT values of -500 to -100 Hounsfield units had an efficacy of $87.5 \%$. By contrast, the localization efficacy in Control Group was $20.0 \%(p=0.032)$. A single surgeon without prior experience performed intraoperative ultrasonography in 20 cases, and the latter 10 procedures required significantly less time relative to the former 10 procedures $(\mathrm{p}=0.000)$. Conclusions: During RATS, the use of intraoperative ultrasonography as an "alternative finger" to "touch" and find the accurate location of pulmonary nodules, especially mixed ground-glass nodules, is warranted.

Keywords: intraoperative ultrasonography; robotics; pulmonary nodule; ground-glass nodule
\end{abstract}

\section{Introduction}

Currently, lung cancer is the most common cancer worldwide and is associated with the highest incidences of cancer-related morbidity and mortality [1-4]. In various populations, the increasing popularity of physical examinations and improvements in health care awareness

Received 22.03.2017 Accepted 11.06.2017

Med Ultrason

2017, Vol. 19, No 4, 374-379

Corresponding author: Jian $\mathrm{Hu}$

Department of Thoracic Surgery, the First Affiliated Hospital, College of Medicine, Zhejiang University,

79 Qingchun Road, Shangcheng District,

Hangzhou, 310003, China

Phone: +8613750801974

Email: dr_hujian@zju.edu.cn have led to tremendous changes in the epidemiology of lung cancer (e.g., prevalence and prognosis) [5]. Currently, chest computed tomography (CT) is used as a primary means of lung cancer screening, and has been credited as contributing to $20 \%$ of the decrease in lung cancer-related mortality related to early lung lesion detections $[6,7]$. Additionally, surgical resection has become the preferred treatment for lung cancer, follow-up studies demonstrating that surgical treatment will maximize the quality of life of patients with non-advanced non-small cell lung cancer (NSCLC) [8,9].

Ultimately, the widespread application of videoassisted thoracoscopy brought lung surgery into the minimally invasive era. The advent of the da Vinci robotic surgical system has provided novel opportunities for the development of minimally invasive thoracic surgery procedures [10-14]. However, the development and 
maturation of this new robotic surgical system cannot be considered apart from other relevant technology [12]. At present, the lack of tactile feedback while using this robotic system remains a major disadvantage because surgeons are unable to accurately locate lesions (e.g., small nodules) with a finger or device, as they would during in video-assisted thoracic surgery (VATS). The resulting pathological uncertainty will have a detrimental effect on subsequent treatment, especially if a resected lesion cannot be located in vitro. However, an invasive technique such as CT-guided hook-wire localization would not be suitable for all cases, as such procedures would be highly risky if the nodule is near the diaphragm, mediastinum or scapula [15-19]. This scenario thereby provides a new opportunity to implement intraoperative ultrasonography as a noninvasive and repeatable localization technique during robotic-assisted pulmonary surgery [20].

A few non-controlled studies with small sample sizes have reported the application of ultrasonography during robotic-assisted thoracic surgery (RATS) [21,22]. Given its characteristics of noninvasiveness, real-time feedback and repeatability, intraoperative ultrasonography could provide valuable pathologic information based on morphological features such as the shape, edge, echo level, blood flow, acoustic shadow and compression simultaneously with lesion location [23,24]. Notably, these benefits have already been confirmed in other areas of surgery.

Accordingly, we conducted this prospective, randomized controlled study to explore the application of ultrasonography for lesion localization during RATS. This study therefore aimed to investigate the effectiveness and applicability of intraoperative ultrasonography for the localization of small pulmonary nodules, and to clarify the relationship between ultrasonography and existing, commonly used means of localization.

\section{Material and methods}

The study was conducted in compliance with the principles of the Declaration of Helsinki. The protocol was approved by the Ethics Committee of the First Affiliated Hospital, College of Medicine, Zhejiang University (2017/104). Written informed consent was obtained from all participants during preoperative communication.

\section{Patients}

Eligible patients included those with CT scan-identified peripheral pulmonary nodules, with no history of thoracic surgery, and who met the criteria for RATS resection. Patients with pulmonary nodules larger than 3 $\mathrm{cm}$ in diameter were excluded from the present study. The eligible patients were prospectively enrolled after providing written informed consent.

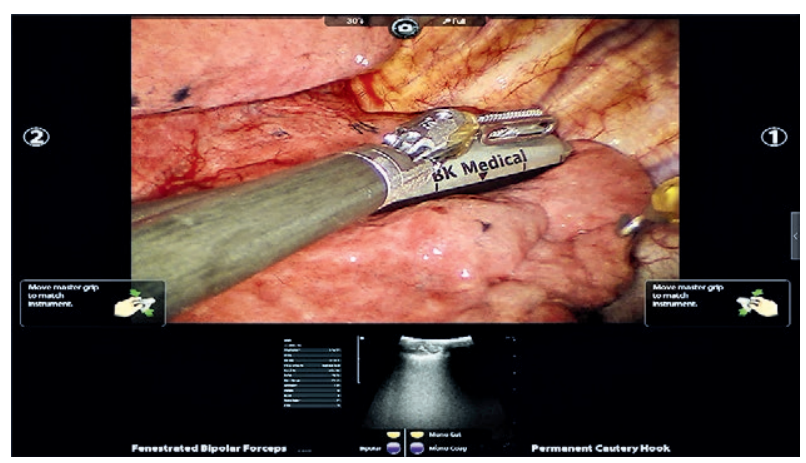

Fig 1. Ultrasonographic images from the surgeon's viewpoint.

\section{Devices and settings}

A newly developed robotic dedicated convex array ultrasound probe (ProART-8826, Brüel \& Kjær Sound \& Vibration Measurement A/S, Skodsborgvej 307, DK2850 Nærum, Denmark) and color Doppler ultrasound scanning diagnostic apparatus (flexFocus 800 ) were used for this study.

The ultrasound devices were connected to the robot console using a DVI signal cable. After equipment placement, the surgeon physician logged in, selected the "Video" menu and "Display Preferences" submenu, and switched the "TilePro" option from "OFF" to "3D". This allowed the ultrasonographic images to be presented synchronously in 3D mode below the original surgical field, as shown in figure 1 .

\section{Study protocol}

All procedures were performed while the patient was under general anesthesia with single lung ventilation through a double-lumen endotracheal intubation.

In the Experimental Group, the ultrasound probe was initially fed into the chest through the auxiliary hole by the assistant surgeon. The probe was then fixed with a bipolar clamp and moved along the pulmonary surface with a slightly more force by the chief surgeon. Another assistant surgeon measured the following ultrasonographic features of nodules that appeared on images: long diameter, echogenicity, border sharpness and sound shadow. Indispensable on-site guidance was provided by an experienced sonologist during the initial stage of the study. Cases in the Experimental group that did not contain detectable nodules of interest were transferred to the Control Group.

In the Control Group, the approximate nodule location was determined by surgeons who had experience with preoperative $\mathrm{CT}$ images.

In both groups, RATS resection was performed after ultrasonographic attempts or empirical localization.

All RATS procedures were performed or supervised by a single thoracic surgeon who had no formal experience with ultrasonography. 


\section{Data collection}

Preoperative patient data (age and sex), surgical parameters (lesion distribution, operation time, and estimated blood loss), localization parameters (duration and outcome), and postoperative hospital data (drainage time, length of stay, and postoperative complications) were collected.

\section{Statistical analysis}

Experimental data were deposited into a database using Microsoft Excel (Microsoft Corporation, Redmond, WA, USA). All statistical calculations were performed using SPSS 20.0 software (IBM Corporation, Armonk, NY, USA). Measurement data were compared using the Student's t-test or the Mann-Whitney U test, and discrete data were compared using the chi-square test or Fisher's exact test. Differences were considered statistically significant at a $p$ value $<0.05$. Figures were drawn using GraphPad Prism 5.0 software (GraphPad Software Inc., La Jolla, CA, USA).

\section{Results}

\section{Baseline characteristics}

Thirty-four patients were enrolled in the present study over 9 operation days. The patients included 17 women and 17 men with ages ranging from 19 to 80 years. Each patient had one pulmonary nodule. The patients were randomly distributed into the Experimental Group and Control Groups, which did not differ significantly in terms of age, sex or lesion distribution (Table I).

\section{Safety verification}

In the Experimental Group, 2 - 15 minutes were required for ultrasonography, and no related complications were observed. The operation time ranged from 44 to 237 min, and the estimated blood loss ranged from 5 to 50 $\mathrm{ml}$. The drainage time and lengths of hospital stay ranged from 2 to 11 days and from 3 to 16 days, respectively.

Table I. Demographic characteristics and distribution of nodules

\begin{tabular}{llll}
\hline & $\begin{array}{l}\text { Experimen- } \\
\text { tal group } \\
(\mathbf{n}=\mathbf{1 7})\end{array}$ & $\begin{array}{l}\text { Control } \\
\text { group } \\
(\mathbf{n}=17)\end{array}$ & p-value \\
\hline Age (years) mean \pm SD & $55.5 \pm 11.9$ & $55.8 \pm 9.7$ & 0.937 \\
Sex-male & 10 & 7 & 0.254 \\
$\begin{array}{l}\text { Distribution } \\
\quad \text { Left upper lobe }\end{array}$ & 3 & & 0.513 \\
$\quad$ Left lower lobe & 4 & 4 & \\
$\quad$ Right upper lobe & 2 & 3 & \\
$\quad$ Right middle lobe & 2 & 5 & \\
Right lower lobe & 6 & 2 & \\
\hline
\end{tabular}

SD - standard deviation
One patient developed postoperative chylothorax and recovered after conservative treatment.

In the Control Group, the estimated blood loss ranged from 20 to $100 \mathrm{ml}$. The operation time, drainage time and lengths of hospital stay ranged from 59 to $140 \mathrm{~min}, 3$ to 12 days, and 4 to 13 days respectively. No patients developed postoperative complications.

None of these differences were statistically significant except for a significantly lower blood loss in Experiment Group, as shown in Table II.

\section{Outcomes of localization}

We attempted to locate a total of 34 nodules in the two groups, and successfully located $13 / 17$ nodules in the Experiment Group and 10/17 in the Control Group. The remaining nodules were confirmed in vitro after wedge resection according to the anatomical location. The analyzed outcomes included the tumor long diameter, distance from pleura, and CT value on the preoperative CT scan (Table III).

Table II. Safety parameters associated with intraoperative localization.

\begin{tabular}{llll}
\hline & $\begin{array}{l}\text { Experimen- } \\
\text { tal group } \\
(\mathbf{n}=\mathbf{1 7})\end{array}$ & $\begin{array}{l}\text { Control } \\
\text { group } \\
(\mathbf{n}=\mathbf{1 7})\end{array}$ & p-value \\
\hline $\begin{array}{l}\text { Surgery duration (min), } \\
\text { mean } \pm \text { SD }\end{array}$ & $92 \pm 44$ & $99 \pm 25$ & 0.591 \\
$\begin{array}{l}\text { Blood loss (ml), } \\
\text { median }\end{array}$ & 89 & 104 & $0.041^{*}$ \\
$\begin{array}{l}\text { Drainage time (days), } \\
\text { mean } \pm \text { SD }\end{array}$ & $4.5 \pm 2.8$ & $4.6 \pm 2.3$ & 0.895 \\
$\begin{array}{l}\text { Hospital stay (days), } \\
\text { mean } \pm \text { SD }\end{array}$ & $5.8 \pm 3.6$ & $6.9 \pm 2.4$ & 0.289 \\
$\begin{array}{l}\text { Complications (Chylo- } \\
\text { thorax })\end{array}$ & 1 & 0 & 1.000 \\
\hline SD - standard deviation & & & \\
\hline
\end{tabular}

Table III. Outcomes of intraoperative localization and related factors.

\begin{tabular}{clll}
\hline & $\begin{array}{l}\text { Experimental } \\
\text { Group }\end{array}$ & $\begin{array}{l}\text { Control } \\
\text { Group }\end{array}$ & P-value \\
\hline $\begin{array}{c}\text { Nodules confirmed } \\
\text { Number (Total) }\end{array}$ & $13(17)$ & $10(17)$ & 0.271 \\
$\begin{array}{c}\text { Distance from Pleura } \\
\text { Within } 1 \mathrm{~cm}\end{array}$ & $11(11)$ & $9(10)$ & 0.476 \\
$\quad$ Within $2 \mathrm{~cm}$ & $13(14)$ & $10(15)$ & 0.169 \\
Long Diameter on CT & & & \\
$\quad$ Within 1 cm & $3(4)$ & $2(6)$ & 0.524 \\
$\quad$ Within 2 cm & $10(13)$ & $7(14)$ & 0.236 \\
CT Value & & & \\
-500 HU and lower & $0(1)$ & $0(3)$ & \\
-500 to-100 HU & $7(8)$ & $1(5)$ & $0.032 *$ \\
-100 HU and higher & $6(8)$ & $9(9)$ & 0.206 \\
\hline
\end{tabular}

CT-computed tomography, HU-Hounsfield unit 


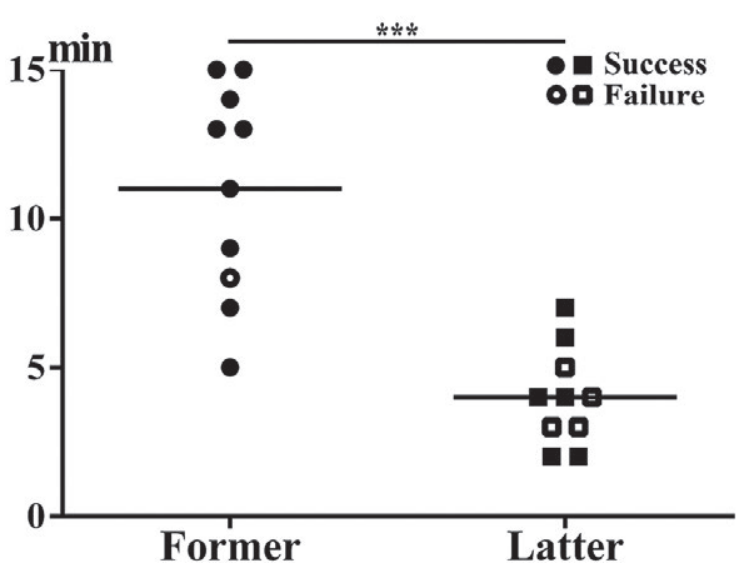

Fig 2. Time required for intraoperative localization.

\section{Time required for intraoperative ultrasonography}

Between a pilot study and the current former study, a single surgeon without former experience performed intraoperative ultrasonography in 20 cases. Significantly less time was required to perform ultrasonography in the latter 10 cases, compared with the former 10 cases (fig 2). In addition, the use of intraoperative ultrasonography did not prolong the operation time in the Experimental Group.

\section{Discussions}

Although ultrasonographic applications are not novel with respect to surgical clinical practice, the lung, a gasbearing tissue, has been "restricted" from the traditional viewing methods used by most surgeons, including thoracic surgeons [25]. Furthermore, experienced surgeons could achieve relatively satisfactory localization using touch during traditional thoracotomy and VATS procedures, thus, reducing the demand for ultrasonographic techniques [21]. In addition, the role and value of ultrasonography in pulmonary surgery remain controversial $[26,27]$. In summary, the application of intraoperative ultrasonography during pulmonary surgery has not yet received sufficient attention or undergone extensive development.

The introduction of the Da Vinci robotic surgical system has created new potential applications for pulmonary ultrasonography. RATS, the result of an inevitable trend of development is similarly complicated by a lack of tactile feedback. Accordingly, thoracic surgeons now require an intraoperative localization method to replace the traditional finger-based tactile procedure. The present study aimed to address this clinical problem by implementing intraoperative ultrasonography to serve as an "alternative finger" during RATS and thus compensate for deficiencies in localization and guidance for followup clinical decisions.

Our study has fully validated the safety of intraoperative ultrasonography in RATS. Specifically, patients in the Experimental Group did not experience an increased operation time, estimated blood loss, postoperative drainage time, or length of hospital stay. Once the relevant technology had been mastered, each intraoperative ultrasonographic procedure required only approximately 4 minutes. Additionally, intraoperative ultrasonography not only improved the accuracy of nodule localization, but also provided information about nodule pathology [22]. Accordingly, surgeons were able to reduce trauma and operation time by clear targeting, accurate lesion resection, and easy verification in isolated tissues.

A CT value of - $100 \mathrm{HU}$ is considered a typical clinical soft tissue value [28]. Pulmonary nodules with CT values exceeding this value are generally considered to be solid pulmonary nodules (SPN), which are not difficult to find intraoperatively. Correspondingly, nodules with CT values less than -100 HU are considered subsolid pulmonary nodules (SSPN). This latter category includes pure ground-glass nodules (pGGN) with CT values less than $-500 \mathrm{HU}$, which are difficult to identify intraoperatively in vivo, and mixed ground-glass nodules (mGGN) with CT values between -500 HU and -100 HU, which are easily palpated.

The results of our study demonstrated that SPNs were generally located with a high level of accuracy, which did not differ significantly between the groups. In contrast, the use of intraoperative ultrasonography led to a significantly better ability to locate mGGNs in Experimental Group, thus demonstrating the usefulness of this "alternative finger".

Although intraoperative ultrasonography during RATS can be performed quickly, safely, noninvasively, accurately, and repeatably, the need for a single ultrasonographic specialist would require too much additional time and expenditure and would counteract the listed advantages. We found the performance of intraoperative ultrasonography by one thoracic surgeon within the team to be the optimal application. Our findings demonstrated that experience with 20 cases was sufficient for the research team to master the basic performance of intraoperative ultrasonography during RATS. Furthermore, the latter 10 cases required significantly less performance time than did the former 10 cases. We must note some inevitable insufficiencies of this preliminary exploratory research study. It was difficult to quantify the advantage of accuracy in the Experimental Group because intraoperative ultrasonographic localization was so accurate in practice, whereas empirical localization was ambiguous. 
Furthermore, as the main endpoint was an initial assessment of the value of intraoperative ultrasonography for lesion localization during RATS, the sample size was not sufficiently large to analyze the ultrasonographic properties and thus infer the pathological nature of nodules $[8,29]$. A semi-quantitative diagnostic model, such as a nomogram, should be established to determine whether a lesion is benign or malignant, based on the ultrasonographic properties of a particular nodule [30].

\section{Conclusions}

In this study, we confirmed that intraoperative ultrasonography could act as an "alternative finger" with which to accurately localize pulmonary nodules, especially mixed ground-glass nodules, during RATS. These findings support the worthiness of this technique and warrant further studies to explore its use for detecting the pathological properties of these nodules.

Acknowledgements: This study was supported by the Major Science and Technology Projects of Zhejiang Province (grant no. 2014C03032).

Conflicts of interest: None to declare.

\section{References}

1. Optican RJ, Chiles C. Implementing lung cancer screening in the real world: opportunity, challenges and solutions. Transl Lung Cancer Res 2015;4:353-364.

2. Siegel RL, Miller KD, Jemal A. Cancer statistics, 2015. CA Cancer J Clin 2015;65:5-29.

3. Chen W, Zheng R, Baade PD, et al. Cancer statistics in China, 2015. CA Cancer J Clin 2016;66:115-132.

4. Siegel RL, Miller KD, Jemal A. Cancer Statistics, 2017. CA Cancer J Clin 2017;67:7-30.

5. Field JK, van Klaveren R, Pedersen JH, et al. European randomized lung cancer screening trials: Post NLST. J Surg Oncol 2013;108:280-286.

6. Henschke CI, Boffetta P, Yankelevitz DF, Altorki N. Computed tomography screening: the international early lung cancer action program experience. Thorac Surg Clin 2015;25:129-143.

7. Heuvelmans MA, Vliegenthart R, Oudkerk M. Contributions of the European trials (European randomized screening group) in computed tomography lung cancer screening. J Thorac Imaging 2015;30:101-107.

8. Schwartz RM, Yip R, Olkin I, Sikavi D, Taioli E, Henschke C. Impact of surgery for stage IA non-small-cell lung cancer on patient quality of life. J Community Support Oncol 2016; 14:37-44.

9. Hong QY, Wu GM, Qian GS, et al. Prevention and management of lung cancer in China. Cancer 2015;121 Suppl 17:3080-3088.
10. Ye X, Xie L, Chen G, Tang JM, Ben XS. Robotic thoracic surgery versus video-assisted thoracic surgery for lung cancer: a meta-analysis. Interact Cardiovasc Thorac Surg 2015;21:409-414.

11. Brooks P. Robotic-assisted thoracic surgery for early-stage lung cancer: a review. AORN J 2015;102:40-49.

12. Ng AT, Tam PC. Current status of robot-assisted surgery. Hong Kong Med J 2014;20:241-250.

13. Park BJ. Robotic lobectomy for non-small cell lung cancer: long-term oncologic results. Thorac Surg Clin 2014;24:157162.

14. Ambrogi MC, Fanucchi O, Melfi F, Mussi A. Robotic surgery for lung cancer. Korean J Thorac Cardiovasc Surg 2014;47:201-210.

15. Mayo JR, Clifton JC, Powell TI, et al. Lung nodules: CTguided placement of microcoils to direct video-assisted thoracoscopic surgical resection. Radiology 2009;250:576585.

16. Chen X, Wang S, Hao Z, Ma Q. Wire 'missing': a rare presentation of preoperative localization wire system dislocation. J Cardiothorac Surg 2014;9:162.

17. Iguchi T, Hiraki T, Gobara H, Fujiwara H, Yamamoto H, Kanazawa S. Tension pneumopericardium as a complication of preoperative localization of a small pulmonary metastasis using a short hook wire and suture system. Cardiovasc Intervent Radiol 2015;38:1346-1348.

18. Hanauer M, Perentes JY, Krueger T, et al. Pre-operative localization of solitary pulmonary nodules with computed tomography-guided hook wire: report of 181 patients. J Cardiothorac Surg 2016;11:5.

19. Ichinose J, Kohno T, Fujimori S, Harano T, Suzuki S. Efficacy and complications of computed tomography-guided hook wire localization. Ann Thorac Surg 2013;96:12031208.

20. Khereba M, Ferraro P, Duranceau A, et al. Thoracoscopic localization of intraparenchymal pulmonary nodules using direct intracavitary thoracoscopic ultrasonography prevents conversion of VATS procedures to thoracotomy in selected patients. J Thorac Cardiovasc Surg 2012;144:1160-1165.

21. Wada H, Anayama T, Hirohashi K, et al. Thoracoscopic ultrasonography for localization of subcentimetre lung nodules. Eur J Cardiothorac Surg 2016;49:690-697.

22. Liu C, Pu Q, Liu L. Is intracavitary thoracoscopic ultrasonography really needed for every intraparenchymal pulmonary nodule? J Thorac Cardiovasc Surg 2013;145:1151.

23. Serrano OK, Huang K, $\mathrm{Ng} \mathrm{N}$, et al. Correlation between preoperative endoscopic ultrasound and surgical pathology staging of gastric adenocarcinoma: A single institution retrospective review. J Surg Oncol 2016; 113:42-45.

24. Becker BA, Fields WA, Pfisterer L, Stuntz RM, Stahlman BA, Kochert EI. Extrabiliary pathology identified by right upper quadrant abdominal ultrasound in emergency department patients. J Emerg Med 2016;50:92-98.

25. Uramoto H, Nakajima Y, Ohtaki K, Kinoshita H. Intraoperative ultrasound elastography has little diagnostic benefit for deeper tumours of the lung. Eur J Cardiothorac Surg 2016;49:1538-1539. 
26. Anayama T, Nakajima T, Dunne M, et al. A novel minimally invasive technique to create a rabbit VX2 lung tumor model for nano-sized image contrast and interventional studies. PLoS One 2013;8:e67355.

27. Kondo R, Yoshida K, Hamanaka K, et al. Intraoperative ultrasonographic localization of pulmonary ground-glass opacities. J Thorac Cardiovasc Surg 2009;138:837-842.

28. Henderson RE, Walker BF, Young KJ. The accuracy of diagnostic ultrasound imaging for musculoskeletal soft tissue pathology of the extremities: a comprehensive review of the literature. Chiropr Man Therap 2015;23:31.

29. Hiller KN, Hagberg CA. Intraoperative pulmonary ultrasound. Anaesth Intensive Care 2014;42:141-142.

30. Juricic J, Ilic N, Frleta Ilic N, Ilic D, Mrklic I, Pogorelic Z. Improved staging using intraoperative ultrasound for mediastinal lymphadenectomy in non-small lung cancer surgery. Surgeon 2014;12:191-194. 